The AsTrophysiCal JoURNAL, 381 :L1-L4, 1991 November 1

(C) 1991. The American Astronomical Society. All rights reserved. Printed in U.S.A.

\title{
THE EVOLUTION OF THE DIFFUSE COSMIC ULTRAVIOLET BACKGROUND CONSTRAINED BY HUBBLE SPACE TELESCOPE OBSERVATIONS OF 3C 273
}

\author{
SATORU IKEUCHI \\ National Astronomical Observatory, Mitaka, Tokyo 181, Japan \\ AND \\ EDWIN L. TURNER \\ Princeton University Observatory, Princeton, NJ 08544 \\ Received 1991 July 8; accepted 1991 August 12
}

\begin{abstract}
Recent Hubble Space Telescope UV spectroscopy of 3C 273 has revealed more low-redshift Lyman- $\alpha$ absorption lines (IGM clouds) than expected from the extrapolation from high-redshift $(z \geq 1.6)$ observations. On the basis of the standard pressure confined cloud model of the Lyman- $\alpha$ forest, we show that this result indicates a sharp drop in the diffuse cosmic UV background from $z=2$ to $z=0$. If we fit a simple power law in $1+z$, the UV background flux must evolve as $(1+z)^{2.7 \pm 1}$. Applying this conclusion to a simple model for a photoionized diffuse component of the IGM, we predict that the H I optical depth (Gunn-Peterson effect) will also only drop slowly or perhaps even increase with decreasing redshift at $z<2$. The implied constraints on the density and pressure of the diffuse IGM at $z=0$ are also derived. We note that the inferred evolution of the diffuse UV flux bears a striking, and perhaps not coincidental, resemblance to the most recent direct determinations of the volume emissivity of the quasar population. Finally, we point out that information on the evolution of the UV flux carries interesting consequences for other cosmological issues such as galaxy formation; in particular, it seems to mitigate somewhat against relatively recent galaxy formation.
\end{abstract}

Subject headings: cosmology — galaxies: intergalactic medium — quasars — ultraviolet: spectra

\section{INTRODUCTION}

The first reports of high-resolution UV spectra of $3 \mathrm{C} 273$ by the Hubble Space Telescope (HST) give very interesting information on the physical nature of the low-redshift universe (Bahcall et al. 1991; Morris et al. 1991): more numerous Ly $\alpha$ absorption lines than predicted by extrapolation from highredshift observations, and a new limit on the depression of the continuum below the Ly $\alpha$ emission line (G-P test; Gunn \& Peterson 1965). Until now such observations have been limited to the high-redshift universe $(z>1.6$ for Ly $\alpha$ lines) or lowresolution spectroscopy.

Numerous, sharp Ly $\alpha$ absorption lines at $z>1.6$ are called the Lyman-alpha forest, which shows a rapid evolution out to $z \gtrsim 3.9$. Based on observed column densities and physical sizes, it is generally accepted that $\mathrm{Ly} \alpha$ absorptions arise from intergalactic clouds with gas mass $\sim 10^{7-8} M_{\odot}$, which are confined by the external pressure of the intergalactic medium (IGM) (Sargent et al. 1980; Ostriker \& Ikeuchi 1983) or by the gravity of cold dark matter (Rees 1986; Ikeuchi 1986). The rapid evolution of detected numbers per unit redshift is usually attributed to the expansion of IGM and/or the evolution of diffuse UV flux, which ionizes and heats the clouds. Therefore, the number density evolution of the $\operatorname{Ly} \alpha$ forest from $z \sim 0.0$ (3C 273) to $z \sim 3.9$ allows exploration of the evolutions of the IGM and the diffuse UV flux. Detection of many more Ly $\alpha$ lines in 3C 273 (Morris et al. 1991) than predicted by extrapolation from high-redshift quasars severely constrains the evolution of the diffuse UV flux, as shown below.

At the same time, this diffuse UV flux ionizes the IGM, which is constrained by the G-P test and by the measurement of the Compton $y$-parameter of the cosmic background radiation (CBR). For the G-P test, the most stringent observed upper limit on $\mathrm{H}_{\mathrm{I}}$ opacity is given as $\tau_{\mathrm{HI}}<0.02$ at $\langle z\rangle=2.64$ by Steidel \& Sargent (1987). The $H S T$ data indicate $\tau<0.04$ at $z=0.158$ (Bahcall et al. 1991). These two results constrain the physical nature of the IGM at two different epochs. Moreover, the $y$-parameter for inverse Compton scattering is limited to $y<10^{-3}$ by $C O B E$ satellite data (Mather et al. 1990).

Combining the above data and connecting them based upon a simple model for the IGM and Ly $\alpha$ clouds, we can obtain constraints on the redshift evolution of the diffuse UV flux and the IGM. In this Letter we present the first attempt at such an analysis. Miralda-Escudé \& Ostriker (1991) have independently reached similar conclusions from a closely related analysis. Turner \& Ikeuchi (1991) and Fukugita \& Lahav (1991) examine the 3C 273 HST data in the context of a nonzero cosmological constant.

\section{EVOLUTION OF LYMAN- $\alpha$ CLOUDS}

\subsection{Pressure-confined Clouds}

Suppose that the Ly $\alpha$ clouds are confined by the pressure of a pervasive intergalactic gas, and both are ionized by diffuse UV flux. Ly $\alpha$ clouds are assumed to be spherical and homogeneous, for simplicity. The mass distribution of the clouds is assumed to be represented by a power law of index $\delta$, that is,

$$
n_{c}(z, M)=n_{c, 0}(1+z)^{3}\left(M / M_{*}\right)^{-\delta},
$$

where the number of clouds per covolume and per unit mass is assumed constant. The expected number of clouds per unit redshift is written (Bergeron \& Ikeuchi 1990)

$$
\frac{d N_{c}}{d z}=\frac{c}{H_{0}} \frac{(1+z)}{\left(1+2 q_{0} z\right)^{1 / 2}} n_{c, 0} \int_{M_{1}}^{M_{2}} \pi R_{c}^{2}(z, M)\left(\frac{M}{M_{*}}\right)^{-\delta} d M,
$$

where we take the Hubble constant to be $H=H_{0}(1+z) \times$ $\left(1+2 q_{0} z\right)^{1 / 2}, R_{c}(z, M)$ is the radius of a cloud, and $M_{1}$ and $M_{2}$ 
are the lower and upper mass limits of clouds. The number density of Ly $\alpha$ clouds per unit $\mathrm{H}_{\mathrm{I}}$ column density $N(\mathrm{H} \mathrm{I})$ is then proportional to

$$
\frac{d^{2} N_{c}}{d z d N(\mathrm{H} \mathrm{I})} \propto \frac{(1+z)}{\left(1+2 q_{0} z\right)^{1 / 2}} n_{\mathrm{H}}^{-2 / 3} M^{2 / 3-\delta} \frac{d M}{d N(\mathrm{H} \mathrm{I})},
$$

where $n_{\mathrm{H}}$ is the total hydrogen density in a cloud and the cloud radius is estimated to be $R_{c} \propto\left(M / n_{\mathrm{H}}\right)^{1 / 3}$.

Since the Ly $\alpha$ forest is an optically thin system photoionized by diffuse UV flux $J(z)$, the ionization state of hydrogen is determined by

$$
n_{\mathrm{HI}} J G_{\mathrm{H}}=n_{\mathrm{H} \text { II }} n_{e} \alpha(T),
$$

where $G_{\mathrm{H}}$ and $\alpha(T)$ are, respectively, the efficiency of ionization and the recombination rate, which is approximated as $\alpha(T)=$ $\alpha_{0} T^{-3 / 4}$ (Black 1981). Then, equation (3) is rewritten as a function of $N(\mathrm{H} \mathrm{I})=2 R_{c} n_{\mathrm{HI}}$,

$$
\frac{d^{2} N_{c}}{d z d N(\mathrm{H} \mathrm{I})} \propto \frac{(1+z)}{\left(1+2 q_{0} z\right)^{1 / 2}} T^{3(5-3 \delta) / 4} n_{\mathrm{H}}^{5 \delta-9} J^{5-3 \delta} N(\mathrm{H} \mathrm{I})^{4-3 \delta}
$$

where we assume that a cloud is highly ionized, $n_{\mathrm{HI}} \ll n_{\mathrm{H}} \sim$ $n_{\mathrm{H} \mathrm{II}} \sim n_{e}$. For the Ly $\alpha$ forest, the $\mathrm{H}$ I column density distribution is represented by $d N_{c} / d N(\mathrm{H}$ I $) \propto N\left(\mathrm{H}_{\mathrm{I}}\right)^{-\beta}$ with $\beta \simeq 1.7( \pm 0.1)$ (Tytler 1987; Sargent, Steibel, \& Boksenberg 1989; Duncan, Ostriker, Bajtlik 1988), which gives rise to the mass distribution index of Ly $\alpha$ clouds,

$$
4-3 \delta=-\beta \rightarrow \delta=(4+\beta) / 3 \simeq 1.9 .
$$

Now we assume that the evolutions of the diffuse UV flux and the Ly $\alpha$ cloud properties are

$$
J=J_{0}(1+z)^{j}, \quad T=T_{0}(1+z)^{t}, \quad n_{\mathrm{H}}=n_{\mathrm{H}, 0}(1+z)^{x} .
$$

Taking the pressure equilibrium condition between a cloud and the IGM, $n_{\mathrm{H}} T=\widehat{P}_{\mathrm{I}}=\hat{P}_{\mathrm{I}, 0}(1+z)^{p}$, the index of density evolution becomes $x=p-t$, where $\hat{P}_{\mathrm{I}}$ is the pressure of the IGM. Introducing equation (7) into equation (5), the number density evolution per unit redshift follows

$$
\begin{aligned}
\frac{d N}{d z} & \propto \frac{(1+z)^{\gamma_{1}}}{\left(1+2 q_{0} z\right)^{1 / 2}}, \\
\gamma_{1} & =1+\frac{3}{4} t(5-3 \delta)+(p-t)(5 \delta-9)+j(5-3 \delta) \\
& \simeq 1-t+0.5 p-0.7 j,
\end{aligned}
$$

where we take $\delta=1.9$ as in equation (6).

\subsection{Comparison with Observations}

From ground-based observations, the number density evolution of the Ly $\alpha$ forest is usually represented by

$$
\frac{d N}{d z} \propto(1+z)^{\gamma}
$$

where the index $\gamma$ for absorption lines with equivalent width $W \geq 0.3 \AA$ at $z \geq 2$ is given by (Murdoch et al. 1986)

$$
\gamma=2.17 \pm 0.36
$$

or that with $W \geq 0.36 \AA$, taking into account the proximity effect, is given by (Lu, Wolfe, \& Turnshek 1991)

$$
\gamma=2.75 \pm 0.29 \text {. }
$$

The HST observations of 3C 273 (Morris et al. 1991) give

$$
\gamma=0.79 \pm 0.37 \text {, }
$$

for $W \geq 0.05 \AA$ between $z=0.0$ (3C 273) and $z=2.0$.

For the evolution law of the IGM pressure, we examine two alternative cases: adiabatic cooling $(p=5)$ and shock heating $(p=3.6)$ at all $z$ (Ikeuchi \& Ostriker 1986). The above models for Ly $\alpha$ clouds and the IGM predict

$$
\gamma=1-q_{0}+\left(\begin{array}{l}
2.5 \\
1.8
\end{array}\right)-0.7 j-t
$$

where the upper and lower parenthetical values give, respectively, adiabatic and shock heating models. Combining equations (11) and (12) to solve for $j$ at $z<2$ in a $q_{0}=\frac{1}{2}$ cosmology gives

$$
j=\left(\begin{array}{l}
3.2 \\
2.2
\end{array}\right)-1.4 t \pm 0.53
$$

Because cooling rates of either pure $\mathrm{H}-\mathrm{He}$ gas or even slightly heavy element enriched plasmas are very steep functions of temperature in the high-ionization regime (Osterbrock 1974), significant temperature variations are not expected (i.e., $t=0$ ). Furthermore, there is no dramatic change in the observed frequencies, widths, strengths, or ionic states of any type of quasar absorption lines at $z \sim 2$ as might be expected if characteristic temperatures changed or if shock heating became dramatically more or less important. Thus, it is most plausible to attribute the change in $\gamma$ to an approximately 1.4 times larger change in $j$, the scenario which we explore in this Letter.

In Figure 1, we illustrate the relation between $\gamma$ and $j$ and show the allowed ranges of $j$ at $z>2$ and $z<2$ for various $q_{0}, p$ combinations. As shown, the observed index $\gamma$ at $z \gtrsim 2$ can be reproduced so long as $j=0 \sim 1$. This result agrees well with that of Bajtlik, Duncan, \& Ostriker (1988) from a consideration of the proximity effect and of Miralda-Escudé \&

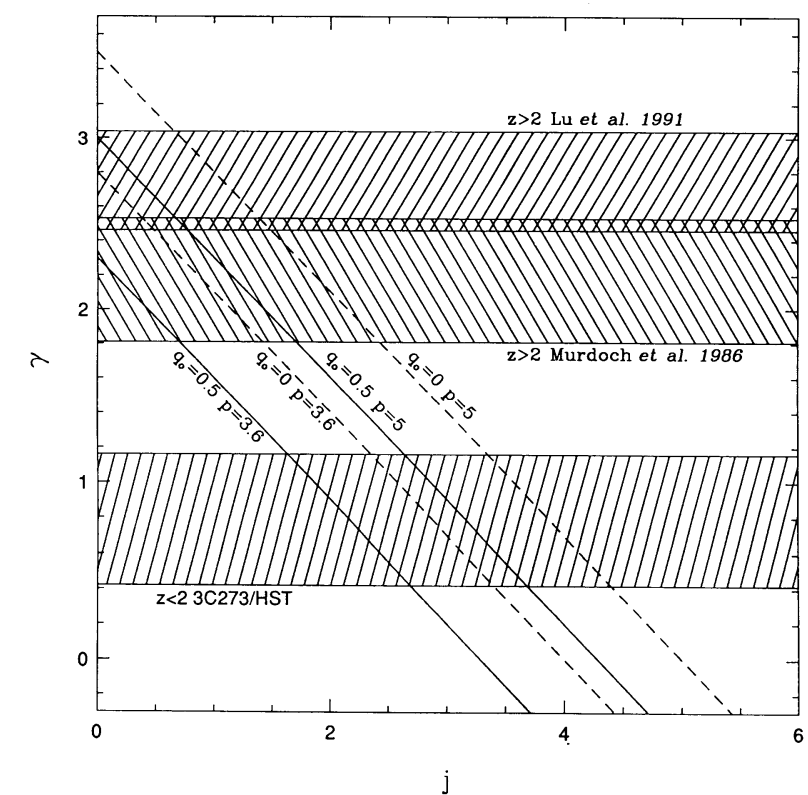

Fig. 1.-Predicted relation between the index $\gamma$ for the number density per unit redshift evolution of the Ly $\alpha$ forest and the index $j$ of the diffuse UV flux. Observed ranges of $\gamma$ at a higher redshift $z>2$ and that estimated from observations of 3C 273 at low $z$ are shown. Shaded regions are allowed. 
Ostriker (1990) from consideration of possible UV sources. Including the present result, three different approaches indicate a slow (or no) evolution of the diffuse UV flux at $z>2$. However, detection of numerous Ly $\alpha$ lines in 3C 273 leads to $\gamma=0.79 \pm 0.37$, if we connect the results at $z \sim 2$ with those for $3 \mathrm{C} 273$. This gives $1.7 \leq j \leq 3.7$. That is, many $\mathrm{Ly} \alpha$ lines can be expected at $0 \leq z \leq 2$ if the diffuse UV flux decreases so rapidly that the $\mathrm{H}_{\mathrm{I}}$ column density is not reduced due to decreasing ionizations.

The scenario of a roughly constant diffuse UV flux at high redshifts suddenly beginning to decrease rapidly toward the present at $z<2$ is perhaps a surprising and not entirely "natural" one. However, the most recent direct determinations of the evolution of the quasar luminosity function (Boyle 1991; Irwin 1991; Schmidt 1991; Warren, Hewett, \& Osmer 1991) indicate that the total quasar UV emissivity rises sharply out to about $z=2$ and then levels off. The best-fit $q_{0}=\frac{1}{2}$ luminosity function (Boyle 1991) has total UV emissivity increasing as $(1+z)^{3.4}$ out to $z=1.9$ and then constant out to redshifts of 3 or greater. The resemblance of this pattern to that inferred above for the UV background based on $L y \alpha$ forest evolution is remarkable and impressive. Of course, it must be admitted that the directly observed quasar population fails by a substantial, though not enormous, factor to supply the absolute level of UV flux apparently required by the available Gunn-Peterson limits and proximity effects (Bechtold et al. 1987; Bajtlik et al. 1988).

Thus, we can conclude that the diffuse UV flux changes from a slow (or no) evolution at $z>2$ to a rapid one at $z<2$. For convenience, we take as a reference model

$$
j=0 \text { at } z>2 \text {, and } j=3 \text { at } z<2 \text {. }
$$

We also consider a variation in which $j=3.4$ at $z \leq 1.9$ as suggested by the quasar observations. The number density evolution per unit redshift for these cases is shown in Figure 2 for $q_{0}=\frac{1}{2}$. The number density evolution per unit redshift shows a sensitive dependence on the index of the IGM pressure, $p$. If $p$ is as large as 5 , the pressure-confined clouds expand so quickly that the detection probability decreases. However, if $p$ is around 3.6, the cloud expansion is relatively small keeping the column density high so that the detectability does not drop much.

\section{INTERGALACTIC MEDIUM}

\subsection{G-P Test and y-Parameter}

The $\mathrm{H}$ I absorption by a pervasive intergalactic gas is well known as the Gunn-Peterson test. The optical depth of $\mathrm{H}$ I gas at the redshift $z$ is given by

$$
\tau_{\mathrm{HI}}(z)=n_{\mathrm{I}, \mathrm{HI}} \sigma_{\mathrm{HI}} \frac{c}{H(z)},
$$

where $\sigma_{\mathrm{HI}}$ is the absorption cross section of UV photons by $\mathrm{H} \mathrm{I}$ gas. At present, only upper limits for $\mathrm{H}_{\mathrm{I}}$ optical depth have been reported. Assuming ionization equilibrium by the diffuse UV flux as in equation (4) and $n_{\mathrm{I}, \mathrm{HI}} \ll n_{\mathrm{I}, \mathrm{H}} \sim n_{\mathrm{I}, \mathrm{H} \text { II }} \sim n_{\mathrm{I}, e}$, the exact expression for the optical depth becomes

$$
\tau_{\mathrm{HI}}(z) \simeq \frac{\sigma_{\mathrm{HI}} c \alpha_{0}}{J_{0} G_{\mathrm{H}} H_{0}} n_{\mathrm{crit}, 0}^{11 / 4} \Omega_{\mathrm{I}, 0}^{11 / 4} \hat{P}_{\mathrm{I}, 0}^{-3 / 4} \frac{(1+z)^{(33-3 p) / 4-j}}{(1+z)\left(1+2 q_{0} z\right)^{1 / 2}},
$$

where $n_{\text {crit, } 0}$ is the critical number density at the present epoch, and the subscript "I" indicates IGM quantities.

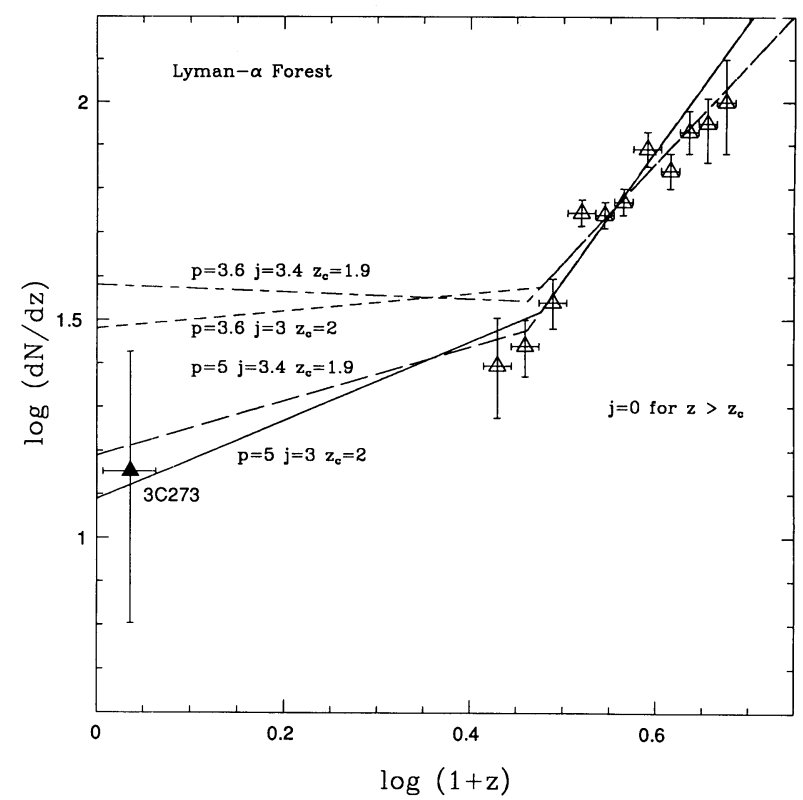

Fig. 2.-Observed (Lu et al. 1991; Morris et al. 1991) and predicted number density of the Ly $\alpha$ forest from $z=3.9$ to $z \sim 0$ (3C 273). Solid and short dashed lines correspond to adiabatic $(p=5)$ and shock heating $(p=3.6)$ models, respectively. Both $j=3$ at $z<2$ reference models and $j=3.4$ at $z<1.9$ models based on quasar observations (long dashes) are shown. High-z data correspond to $W \geq 0.36 \AA$, while the $3 C 273$ point is for $W \geq 0.30 \AA$; thus, a small systematic shift is expected in addition to the statistical errors indicated in the plot.

More information on the IGM is provided by the $y$ parameter of inverse Compton scattering of CBR photons

$$
y=\int_{0}^{z_{i}} \frac{k T_{\mathrm{I}}}{m_{e} c^{2}} \sigma_{\mathrm{T}} n_{\mathrm{I}, e}(z) \frac{c}{H(z)} d z,
$$

where $\sigma_{\mathrm{T}}$ is the cross section of Thomson scattering. For an almost wholly ionized IGM $n_{\mathrm{I}, e} \sim n_{\mathrm{I}, \mathrm{H}}$, we have

$$
y=\frac{\sigma_{\mathrm{T}} c k \hat{P}_{\mathrm{I}, 0}}{2 m_{e} c^{2} H_{0}} \int_{0}^{z_{i}} \frac{(1+z)^{p}}{(1+z)\left(1+2 q_{0} z\right)^{1 / 2}} d z .
$$

\subsection{Comparisons with Observations}

The most severe observational limit to the $\mathrm{H}$ I optical depth is given by Steidel \& Sargent (1987) as $\tau_{\mathrm{HI}}<0.02$ at $\langle z\rangle=2.64$. The HST result indicates $\tau_{\mathrm{HI}}<0.04$ at $z=0.158$ (Bahcall et al. 1991), which is stronger than that given by the IUE observation (Boksenberg et al. 1978). The predicted optical depth in equation (16) must run below these observed limits. Here, we take $H_{0}=100 \mathrm{~km} \mathrm{~s}^{-1} \mathrm{Mpc}^{-1}$ and $J_{0}=$ $10^{-21}(1+z / 3)_{z=0}^{j}$ ergs s${ }^{-1} \mathrm{~cm}^{-2} \mathrm{sr}^{-1} \mathrm{~Hz}^{-1}$. The upper bound from the G-P test at $z=2.64$ is calculated for evolution law of the diffuse UV flux as in equation (14) to be

$$
\begin{aligned}
\Omega_{\mathrm{I}, 0}^{11 / 4} \hat{P}_{\mathrm{I}, 0}^{-3 / 4} & <4.1 \times 10^{-6} \quad(p=5) \\
& <1.1 \times 10^{-6} \quad(p=3.6) .
\end{aligned}
$$

In Figure 3 we show the predicted $\mathrm{H}_{\mathrm{I}}$ optical depth versus redshift. We see that the $\mathrm{H}_{\mathrm{I}}$ optical depth shows a similar behavior to the number density evolution of Ly $\alpha$ lines per unit redshift displayed in Figure 2, but the case of $p=5$ predicts a higher optical depth than that of $p=3.6$. This is easily understood as follows: A rapid decrease of IGM pressure gives rise to a rapid drop of IGM temperature for a fixed density. Thus, 


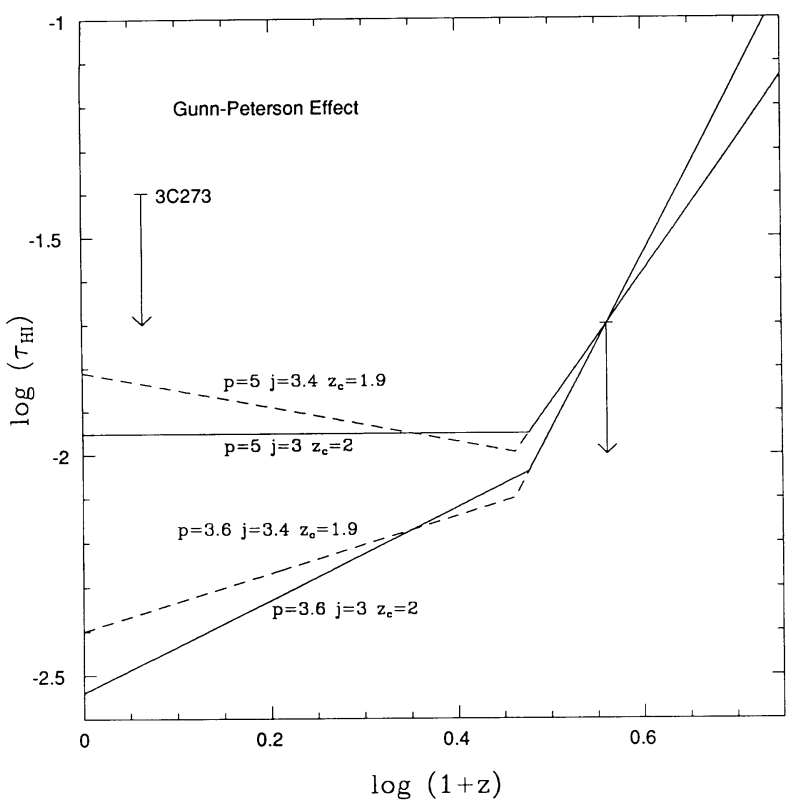

FIG. 3. Calculated upper limit of $\mathrm{H}_{\mathrm{I}}$ optical depth normalized as $\tau_{\mathrm{HI}}=$ 0.02 at $z=2.64$. Both $J=3$ at $z<2$ references models and $j=3.4$ at $z<1.9$ models based on quasar observations are shown using solid and dashed lines, respectively.

the recombination increases and the $\mathrm{H}_{\mathrm{I}}$ optical depth increases even though the IGM is expanding. It is highly desirable to measure the $\mathrm{H}_{\mathrm{I}}$ optical depth not as an upper limit but as a detected finite value. This may be possible by combining data for a sample of low-redshift quasars because it should not decrease or might even increase at $z<2$.

The upper limit on the $y$-parameter of the CBR is given by $C O B E$ (Mather et al. 1990) as $y<10^{-3}$. From equation (18), we get for $q_{0}=\frac{1}{2}$,

$$
\begin{array}{r}
\hat{P}_{1,0}<2.0 \times 10^{3} \frac{\left(p-q_{0}\right)}{\left[\left(1+z_{i}\right)^{p-q_{0}}-1\right]}=2.84 \quad(p=5), \\
=24.1 \quad(p=3.6) .
\end{array}
$$

The upper and lower limits of the IGM pressure determined, respectively, by the $y$-parameter and the G-P test are not so stringent as those obtained from the stability conditions for Ly $\alpha$ clouds by Ikeuchi \& Ostriker (1986).

\section{CONCLUSION AND REMARKS}

Based upon a simple pressure-confined cloud model, we can determine the evolution law of the diffuse UV flux from the detection of numerous Ly $\alpha$ lines in 3C 273: A slow (or no) evolution at $z>2$ and a rapid decrease at $z<2$ are indicated. This conclusion is consistent with other evidence obtained from the proximity effect (Bajtlik et al. 1988), possible UV sources (Miralda-Escudé \& Ostriker 1990), and observed quasar evolution (Boyle 1991). This conclusion can be checked by observing more Ly $\alpha$ lines between $z=0$ and 2 . The expected number of $\mathrm{Ly} \alpha$ lines per unit redshift should not decrease or might even increase with decreasing redshift at $z<2$. From this behavior, the evolution law of the IGM pressure as either adiabatic $(p=5)$ or shock heated $(p=3.6)$ could be determined.

Applying this result to the G-P test, we calculate the expected evolution of the $\mathrm{H}_{\mathrm{I}}$ optical depth from higher to lower redshifts. The $\mathrm{H}$ I optical depth also does not decrease and might even increase at $z<2$. Combining the G-P test and the observed upper limit on the $y$-parameter, we constrain the ranges of pressure and density of the IGM (eqs. [19] and [20]).

Ideally we would like to incorporate information on the IGM and the diffuse UV background into a comprehensive physical model of the universe at moderate redshifts. This task is clearly beyond the scope of the present Letter; however, we may utilize the results of the extensive study of extragalactic UV sources by Miralda-Escudé \& Ostriker (1990) to make a tentative initial exploration of such connections. They predicted ionizing flux as a function of redshift for models in which both quasar and galaxy (i.e., hot stars) sources were considered in addition to various models of extragalactic dust extinction and Lyman continuum absorption. If we accept the conclusion developed in this Letter that the diffuse UV flux has fallen by a factor of 27 (third power of $1+z$ ) since a redshift of 2 , this imposes a significant additional constraint on their models. In particular, their models in which the diffuse UV flux is dominated by the hot stars in forming galaxies give too much ionization at $z=0$ to be consistent with the 3C 273 observations, particularly if the epoch of galaxy formation is relatively recent. Of course, these models were not devised with the new $H S T$ results in mind, and it might be relatively easy to modify the models in order to accommodate them. In any case, this can only be considered an extremely preliminary and qualitative discussion of the topic.

The authors would like to thank Professors J. Bahcall and B. Savage for providing preprints of the HST results and S. L. Morris for valuable criticisms of an earlier version of this Letter. We also thank H. Suzuki for her careful word processing. This work was supported in part by NASA grant NAGW-2448.
Bahcall, J N et al. 1991, preprint

Bajtlik, S., Duncan, R. C., \& Ostriker, J. P. 1988, ApJ, 327, 570

Bechtold, J., Weymann, R. J., Lin, Z., \& Malkan, M. A. 1987, ApJ, 337, 609

Bergeron, J., \& Ikeuchi, S. 1990, A\&A, 235, 8

Black, J. H. 1981, MNRAS, 197, 553

Boksenberg, A., et al. 1978, Nature, 275, 404

Boyle, B. 1991, in Space Distribution of Quasars, ed. D. Crampton (San Francisco: Astron. Soc. of the Pacific), in press

Duncan, R. C., Ostriker, J. P., \& Bajtlik, S. 1988, Princeton preprint

Fukugita, M., \& Lahav, O. 1991, MNRAS, submitted

Gunn, J. E., \& Peterson, B. A. 1965, ApJ, 142, 1633

Ikeuchi, S. 1986, Ap\&SS, 118, 509

Ikeuchi, S., \& Ostriker, J. P. 1986, ApJ, 301, 522

Irwin, M. 1991, in Space Distribution of Quasars, ed. D. Crampton (San

Francisco: Astron. Soc. of the Pacific), in press

Lu, L., Wolfe, A. M., \& Turnshek, D. A. 1991, ApJ, 367, 19

Mather, J. C., et al. 1990, ApJ, 354, L37

Miralda-Escudé, J., \& Ostriker, J. P. 1990, ApJ, 250, 1

\section{REFERENCES}

Miralda-Escudé, J., \& Ostriker, J. P. 1991, ApJ, submitted

Morris, S. L., Weymann, R. J., Savage, B. D., \& Gilliland, R. L. 1991, preprint

Murdoch, H. S., Hunstead, R. W., Pettini, M., \& Blades, J. C. 1986, ApJ, 309, 19

Osterbrock, D. E. 1974, Astrophysics of Gaseous Nebulae (San Francisco: Freeman), 54

Ostriker, J.P. \& Ikeuchi, S. 1983, ApJ, 263, L63

Rees, M. J. 1986, MNRAS, 218, 25P

Sargent, W. L. W., Steidel, C. C., \& Boksenberg, A. 1989, ApJS, 69, 703

Sargent, W. L. W., Young, P. J., Boksenberg, A., \& Tytler, D. 1980, ApJS, 42, 41

Schmidt, M. 1991, in Space Distribution of Quasars, ed. D. Crampton (San Francisco: Astron. Soc. of the Pacific), in press

Steidel, C. C., \& Sargent, W. L. W. 1987, ApJ, 318, L11

Turner, E. L., \& Ikeuchi, S. 1991, ApJ, submitted

Tytler, D. 1987, ApJ, 321, 49

Warren, S., Hewett, P., \& Osmer, P. 1991, in Space Distribution of Quasars, ed. D. Crampton (San Francisco: Astron. Soc. of the Pacific), in press 